\title{
光、电催化硫氰基化反应研究进展
}

\author{
张龙菲牛聪杨晓婷秦宏云 \\ 杨建静文江伟* 王 桦* \\ (曲阜师范大学化学与化工学院 山东曲阜 273165)
}

\begin{abstract}
摘要 硫氰酸酯作为一种用途广泛的合成子, 在医药、农药、材料等诸多领域中具有重要的应用价值. 特别是, 通过光、 电催化硫氰基化反应的合成途径具有绿色、高效、安全等优势，在有机化学中得到广泛关注. 重点介绍了一系列基于 光、电催化的偶联/硫氧基化反应，期望为探究硫氰酸酯类化合物的绿色合成提供帮助.
\end{abstract}

关键词 硫氧化; 光催化; 电催化

\section{Recent Advances on the Photocatalytic and Electrocatalytic Thiocyanation Reactions}

\author{
Zhang, Longfei Niu, Cong Yang, Xiaoting Qin, Hongyun \\ Yang, Jianjing Wen, Jiangwei* Wang, Hua* \\ (Institute of Medicine and Materials Applied Technologies, College of Chemistry and Chemical Engineering, \\ Qufu Normal University, Qufu, Shandong 273165)
}

\begin{abstract}
Thiocyanate, as a versatile synthon, which has important application value in many fields such as pharmaceutical, pesticide and materials. The photocatalytic and electrocatalytic thiocyanation reactions have been widely concerned in organic chemistry due to the advantages of green, efficiency and safety. In this review, the cross-coupling/thiocyanation reactions based on the photocatalytic and electrocatalytic are described, which is expected to be helpful in exploring the green synthesis of thiocyanates compounds.

Keywords thiocyanation; photocatalytic; electrocatalytic
\end{abstract}

有机硫氰酸盐(RSCN)是一种重要的有机合成中间 体，通过简单的化学转换即可获得硫醚、硫醇、异硫氰、 含硫含氮的杂环等多种类型的有机硫化物. 此外, 这些 硫氧酸酯类化合物具有抗微生物活性、抑制酶活性、 杀虫活性等多种生物活性, 在医药、农药和材料等诸 多领域中具有重要应用价值 ${ }^{[1]}$. 优异的生物活性和广阔 的应用前景, 极大地引起了有机合成化学家们的研究兴 趣.

近年来, 有机化学家们对硫氰基化反应及应用开展 了较为深入的研究和探索, 其构建策略主要包括过渡金 属或非金属催化, 或化学计量氧化剂参与的传统硫氰基
化策略(Scheme 1a)，其主要涉及亲核取代、亲电取代和 自由基等反应类型 ${ }^{[2]} .2016$ 年, Gulea ${ }^{[3]}$ 和 Pate ${ }^{[4]}$ 分别对硫 氯酸酯和 $\alpha$-羰基硫氰酸酯类化合物的合成及应用做了 很好的总结. 最近，金城安等 ${ }^{[5]}$ 重点归纳总结了近三年 来硫氰基化合物的合成进展，对硫氰化研究领域的发展 具有重要作用.

开发绿色、高效、高原子经济性的 $\mathrm{C}-\mathrm{S}$ 键构筑策 略是有机合成化学领域重要的研究内容之一 ${ }^{[6]}$. 光催化 是以光能作为直接能源, 电催化是以电能(电子传递)作 为能源, 符合绿色化学要求. 近年来, 有机合成工作者 们利用光催化策略, 以空气作为绿色氧化剂已成功实现

\footnotetext{
* Corresponding authors. E-mail: huawang@qfnu.edu.cn; wenjy@qfnu.edu.cn

Received December 9, 2019; revised January 9, 2020; published online January 21, 2020.

Project supported by the National Natural Science Foundation of China (Nos. 21902083, 21675099), the Major Basic Research Program of Natural Science Foundation of Shandong Province (No. ZR2018ZC0129), the College Students Innovation and Entrepreneurship Training Project of Shandong Province (No. S201910446044) and the Qufu Normal University Research Startup Fund (Nos. 6132, 6125).

国家自然科学基金(Nos. 21902083, 21675099)、山东省重大自然科学基金(No. ZR2018ZC0129)、山东省省级大学生创新创业项目(No. S201910446044) 以及曲阜师范大学科研启动基金(No. 6132, 6125)资助项目.
} 
多种类型的硫氰基化反应. 此外, 有机电化学合成作为 一种新颖的、绿色的策略, 是利用连续不断的电子得失 来替代传统的化学氧化还原试剂. 有机合成工作者利用 这一特性成功地实现了一系列无金属、无氧化剂参与 的硫氰基化反应(Scheme 1b). 在这篇综述中, 整理讨 论了利用光催化、电催化的新型策略来实现硫氧基化 反应，以显示光催化、电催化硫氰基化反应的独特性 和新型性, 以期为读者了解光、电催化硫氧化反应的研 究动态提供帮助.

(a) Traditional thiocyanation

$$
\mathrm{R}^{1-\mathrm{H}}+\mathrm{RSCN} \underset{[\mathrm{O}]}{\stackrel{\text { Metal or metal-free }}{\longrightarrow}} \mathrm{R}^{1}-\mathrm{SCN}
$$

(b) Photo-/electrochemistry-induced thiocyanation

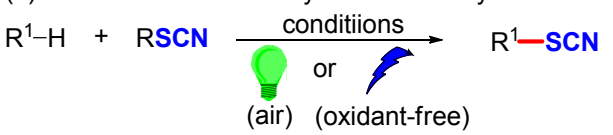

图式 1 硫氰化策略

Scheme 1 Thiocyanation strategy

\section{1 光催化硫氭基化反应}

\section{1 光催化 $\mathrm{C}\left(\mathrm{sp}^{3}\right)-\mathrm{H}$ 的硫氰基化反应}

通过 $\mathrm{C}-\mathrm{H}$ 键活化官能化反应构建 $\mathrm{C}-\mathrm{S}$ 键是最 直接、高效和绿色的途径, 具有原子经济性而被广泛 关注 ${ }^{[5]}$. 由于惰性的 $\mathrm{C}\left(\mathrm{sp}^{3}\right)-\mathrm{H}$ 键的活化往往需要较高 的活化能, 这增加了 $\mathrm{C}\left(\mathrm{sp}^{3}\right)-\mathrm{H}$ 键活化官能化的难度和 挑战. 与传统热力学 $\mathrm{C}\left(\mathrm{sp}^{3}\right)-\mathrm{H}$ 硫氰基化反应方法相比, 光催化氧化偶联 $\mathrm{C}\left(\mathrm{sp}^{3}\right)-\mathrm{H}$ 硫氰基化反应是实现 $\mathrm{C}\left(\mathrm{sp}^{3}\right)$ $-\mathrm{H}$ 参与硫氰基化反应的有效策略.

2015 年, Yadav 课题组 ${ }^{[7]}$ 报道了一种以 Eosin Y 为光 催化剂, 空气氛围中, 在绿光照射下实现叔胺 $\alpha$-位 $\mathrm{C}\left(\mathrm{sp}^{3}\right)-\mathrm{H}$ 键的硫氰基化反应. 该方法具有光催化剂用 量低(Eosin Y 1 mol\%)、反应温度低、底物适用范围宽等 优点. 该方法能够以 74\% 95\%的产率获得一系列硫氧 化四氢异喹啉衍生物 2. 根据研究, 作者提出了一种可 能的机理: 首先, $\mathrm{EY}$ 被光激发到激发态 $\mathrm{EY}^{*}$, 底物与激 发态 $\mathrm{EY} *$ 经单电子转移得到中间体 $\mathbf{A}$ 和 $\mathrm{RB}^{\bullet-}$; 随后, 氧 气氧化 $\mathrm{RB}^{\bullet-}$ 得到基态 $\mathrm{EY}$, 并生成 $\mathrm{O}_{2}{ }^{\bullet-}, \mathrm{O}_{2}{ }^{--}$可筫取 $\mathbf{A}$ 的 $\alpha$ 位质子形成亚胺离子中间体 $\mathbf{B}$; 最后, $\mathrm{SCN}$ 进攻 $\mathbf{B}$ 得 到最终目标产物(Scheme 2).

2018 年, 刘强课题组 ${ }^{[8]}$ 利用苂光素作为光催化剂, 在氧化条件下, 通过交叉偶联硫氰酸铵和活化羰基酮类 化合物, 为氨化/硫氰化烯烃的构建发展了一种简单、高 效、高原子经济的合成方法(Scheme 3). 该方法以低毒、 廉价的硫氰酸铵为胺源和硫氰基源, 可以方便、高效地 获得一系列多取代烯烃, 副产物为水. 作者结合机理实 验和理论计算, 提出了一种可能的机理: 反应开始于荧

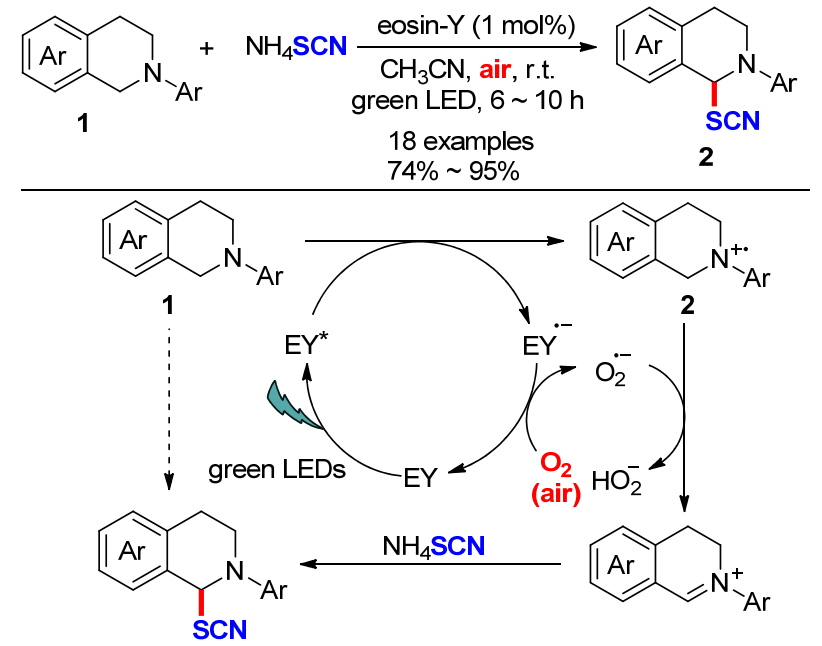

图式 2 叔胺 $\alpha-\mathrm{C}\left(\mathrm{sp}^{3}\right)-\mathrm{H}$ 硫氰化

Scheme $2 \alpha-\mathrm{C}\left(\mathrm{sp}^{3}\right)-\mathrm{H}$ thiocyanation of tertiary amines

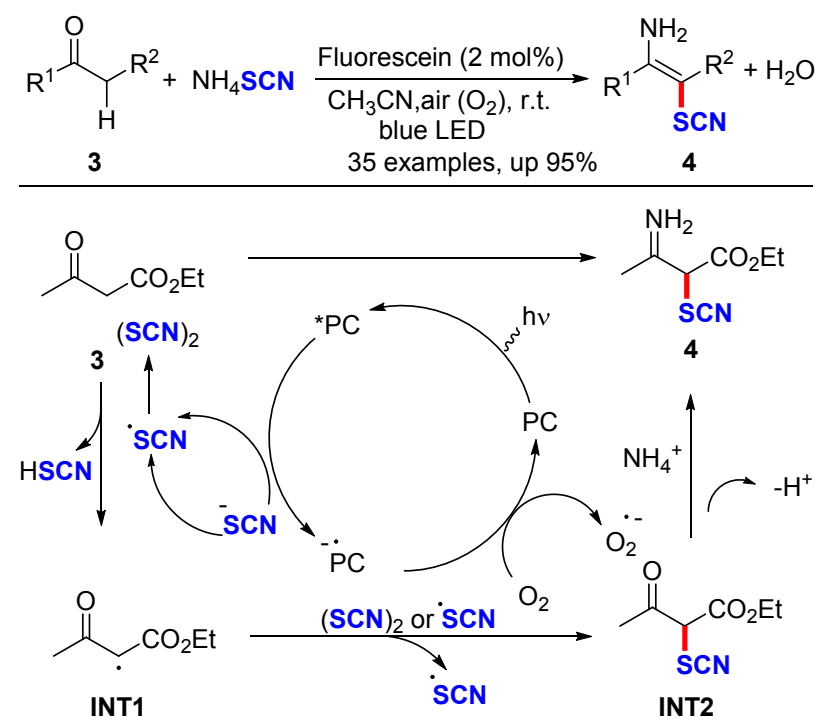

图式 3 活化酮的氨基硫氰化反应

Scheme 3 Aminothiocyanation of activated ketones

光素被可见光照射激发到激发态经单电子氧化硫氰酸 铵生成・SCN 自由基和 $\mathrm{PC}^{-}$, 氧气氧化 $\mathrm{PC}^{\cdot-}$ 生成超氧化 物 $\mathrm{O}_{2}{ }^{-}$并再生光催化剂. 然后, $\cdot \mathrm{SCN}$ 自由基从 3 中瞳氢, 生成碳中心自由基 INT1 和硫氰酸，获得的中间体 INT1 被其它・SCN 捕获生成中间体 INT2. 由于 INT2 中羰基 缺电子容易被 $\mathrm{NH}_{3}$ 亲核进攻，并消除水得到目标产物 4 .

\section{2 光催化 $\mathrm{C}\left(\mathrm{sp}^{2}\right)-\mathrm{H}$ 的硫氭基化反应}

\subsection{1 光催化烯烃的硫氰基化反应}

光催化自由基硫氰基化反应是合成有机硫氰化物 的一种重要途径. 1978 年, Guy 课题组 ${ }^{[9]}$ 就发展了一种紫 外光照射，室温环境下，通过自由基加成途径实现了烯 烃的双硫氰基化反应，该方法能够以 $24 \% \sim 99 \%$ 的收率 获得一系列的双硫氰化烯烃(Scheme 4), 不足是该方法 需要用高能量的紫外光作为光源. 


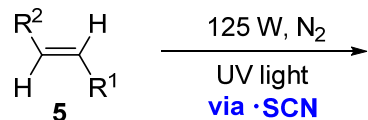

$\mathrm{R}^{1}=\mathrm{H}, \mathrm{Ph}, \mathrm{Me}, \mathrm{Et},{ }^{i} \mathrm{Pr},{ }^{\mathrm{t}} \mathrm{Bu}$

$\mathrm{R}^{2}=\mathrm{H}, \mathrm{Me}, \mathrm{Et},{ }^{i} \mathrm{Pr}$

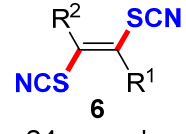

24 examples

$24 \% \sim 99 \%$
图式 4 烯烃双硫氰化反应

Scheme 4 Dithiocyanation of olefins

2015 年, Guan 等 ${ }^{[10]}$ 采用可见光诱导的无金属硫氰 酸酯自由基加成/分子内环化串联反应，以 $N$-烷基- $N$-甲 基丙烯基苯酰胺为原料实现了硫氧化异喹啉二酮类化 合物的合成(Scheme 5). 作者利用吟啶盐作为光催化 剂、利用廉价易得的硫氰酸铵通过单电子氧化得到硫氰 基自由基, 实现了 21 个具有高功能化药物样分子结构 的硫氰化异喹啉二酮类化合物的绿色构建. 作者根据一 系列的机理实验以及相关文献基础上, 提出了一种可能 的反应机理: 首先, 在可见光照射下, $\mathrm{Acr}^{+}-\mathrm{Mes}$ 被光激 发为 $\mathrm{Acr}^{+}-\mathrm{Mes}$, 激发态光催化剂 $\mathrm{Acr}^{+}-\mathrm{Mes}$ *从硫氭酸 盐中篗取一个电子得到 ${ }^{\circ} \mathrm{SCN}$, 同时生成 Acr-Mes. 随 后, Acr-Mes 被氧气氧化为 $\mathrm{Acr}^{+}-\mathrm{Mes}$ 完成光催化循环, 并产生超氧阴离子 $\mathrm{O}_{2}{ }^{-}$拿走 $\mathrm{NH}_{4}{ }^{+}$质子得到 $\mathrm{HO}_{2}{ }^{\circ}$. 同时, 生成的硫氰基自由基与 7 发生自由基加成形成 $\mathrm{C}-\mathrm{S}$ 键, 并得到烷基自由基中间体 $\mathbf{8}, \mathbf{8}$ 经自由基芳构化生成中间
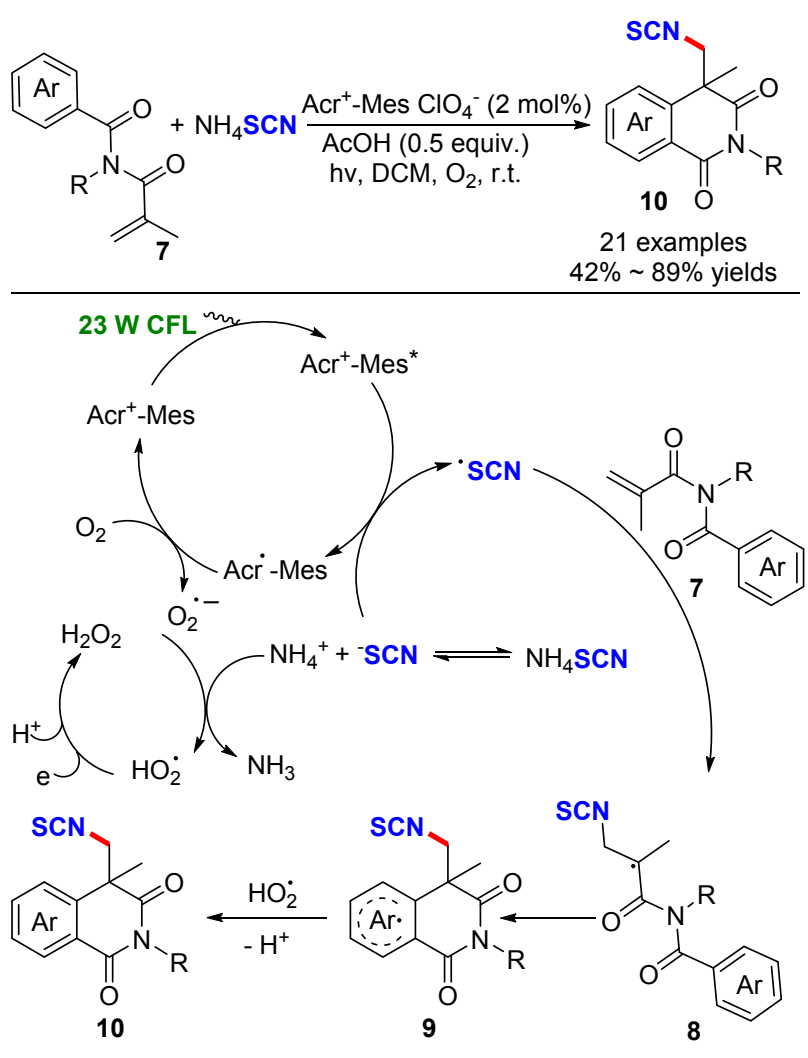

图式 5 光催化活化的烯酰胺衍生物的硫氧化反应 Scheme 5 Photocatalytic thiocyanation of activated enamide derivatives
体 $\mathbf{9}, \mathrm{HO}_{2}$ •氧化 9 得到目标产物 $\mathbf{1 0}$.

2018 年, Bolm 课题组 ${ }^{[11]}$ 以 $\mathrm{N}-\mathrm{Br}$ 亚砜亚胺与 $\mathrm{NH}_{4} \mathrm{SCN}$ 为原料, 以二氯甲烷(DCM)作为溶剂, 在常温 下反应 $16 \mathrm{~h}$ 制备了一种新型硫氰化试剂(11). 这种新型 的硫氰化试剂在蓝光照射下, 以 Ir 为催化剂, 可以很好 地应用于烯烃的双官能团化反应，能够以 $38 \% \sim 81 \%$ 的 收率获得一系列新颖的硫氧化亚胺化产物 13(Scheme $6)$.

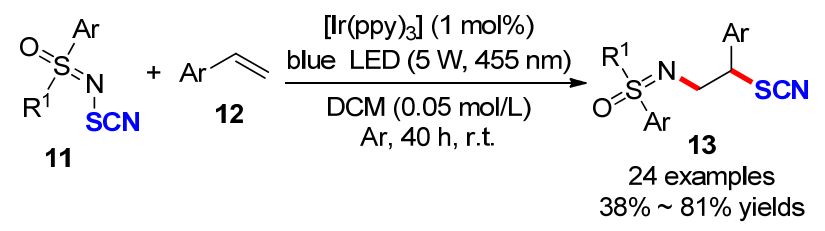

图式 6 光催化烯烃的硫氰化和磺酰胺化反应

Scheme 6 Photocatalytic thiocyanation and sulfonamidation of olefins

2019 年, 万结平课题组 ${ }^{[12]}$ 利用可见光诱导策略成 功实现了叔烯胺酮 $\alpha-\mathrm{C}-\mathrm{H}$ 键活化硫氰化反应. 该方法 为构建硫氰化烯烃类衍生物提供了一种直接、简单、有 效的方法. 作者通过在底物 14 的邻位引入一个羟基官 能团, 实现硫氧化色酮类衍生物 16 的合成. 当将光催化 剂由 Rose Bengal 改为 $\mathrm{Ru}(\mathrm{bpy})_{3} \mathrm{Cl}_{2} \bullet 6 \mathrm{H}_{2} \mathrm{O}$ 时, 能够得到 一系列具有氨基和硫氰基的双官能化烯烃 17 , 作者认 为在该过程中 $\mathrm{Ru}(\mathrm{bpy})_{3} \mathrm{Cl}_{2} \cdot 6 \mathrm{H}_{2} \mathrm{O}$ 的作用为路易斯酸 (Scheme 7).

\subsection{2 光催化芳杂环化合物的硫氧基化反应}

近几年来, 有机化学家们又发展了一系列高效节能 的可见光催化硫氰化策略, 相对传统的紫外光催的硫氰 化反应，该类策略是直接以可见光为光源、空气作为氧 化剂, 具有绿色环保、能耗低的优点. 例如, 2014 年, Li 课题组 ${ }^{[13]}$ 为吲哚 $\beta$ 位引入硫氰基发展了一种绿色、高效 的可见光催化策略(Scheme 8). 以 $14 \mathrm{~W}$ 的节能灯为光 源, 廉价易得的 Rose Bengal 为光催化剂, 空气为氧化 剂, 在四氢呋喃溶剂中照射 $24 \mathrm{~h}$, 以 $73 \% \sim 95 \%$ 的产率 获得 15 个不同种类的 $\beta$-硫氰化吲哚化合物. 作者认为 该反应机理可能是: 首先, 光照 $\mathrm{RB}$ 转变为激发态 $R B *$, $\mathrm{SCN}$ 被 $\mathrm{RB}$ *经单电子氧化得到 ${ }^{\circ} \mathrm{SCN}$ 自由基 $\mathbf{A}$, 并产生 $\mathrm{RB}^{\circ}$ - 自由基阴离子被氧气氧化还原为基态 $\mathrm{RB}$. 随后, $\cdot \mathrm{SCN}$ 自由基 $\mathbf{A}$ 与底物 18 经亲电加成得到中间体 $\mathbf{B}, \mathbf{B}$ 被进一步氧化产生阳离子中间体 $\mathbf{C}$. 最后, C 失去质子 得到目标产物 19.

随后, He 课题组 ${ }^{[14]}$ 借用异相催化策略为吲哚 $\beta$ 位引 入硫氰基发展了一种无毒、易得、稳定的 $\mathrm{TiO}_{2} / \mathrm{MoS}_{2}$ 纳 米光催化剂. 该方法对电中性的底物具有较好的催化效 率，并能获得优异的收率. 然而，该策略在底物的适用 


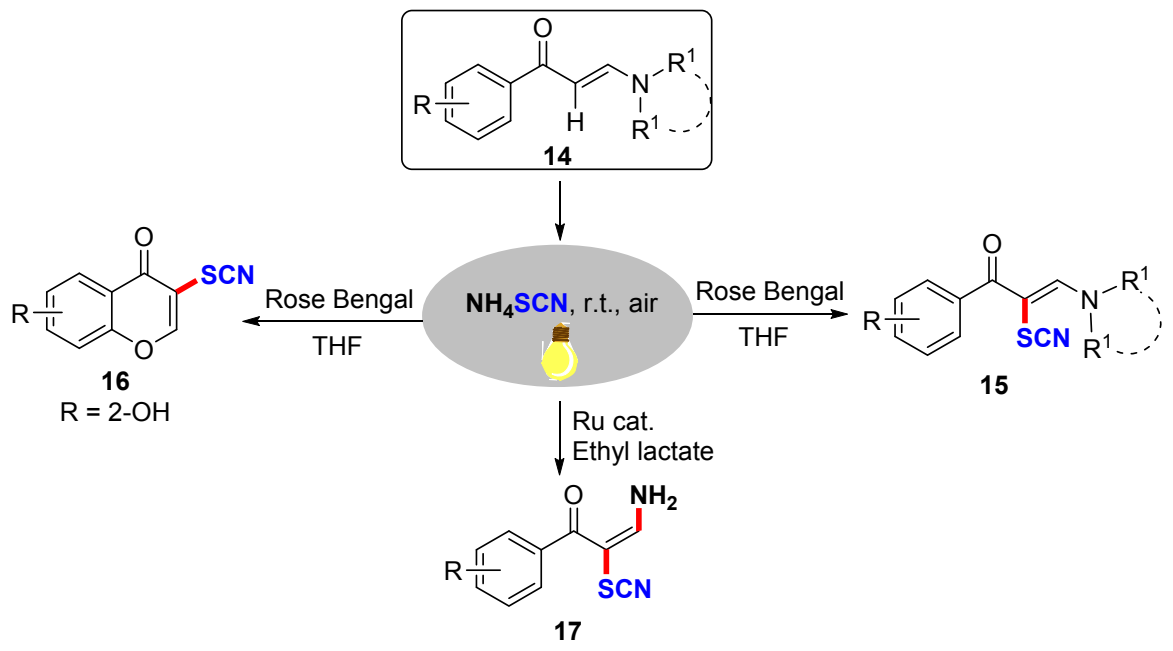

图式 7 叔烯胺酩 $\alpha$-位硫氰化反应

Scheme 7 Thiocyanation on the $\alpha$-site of tertiary enaminones

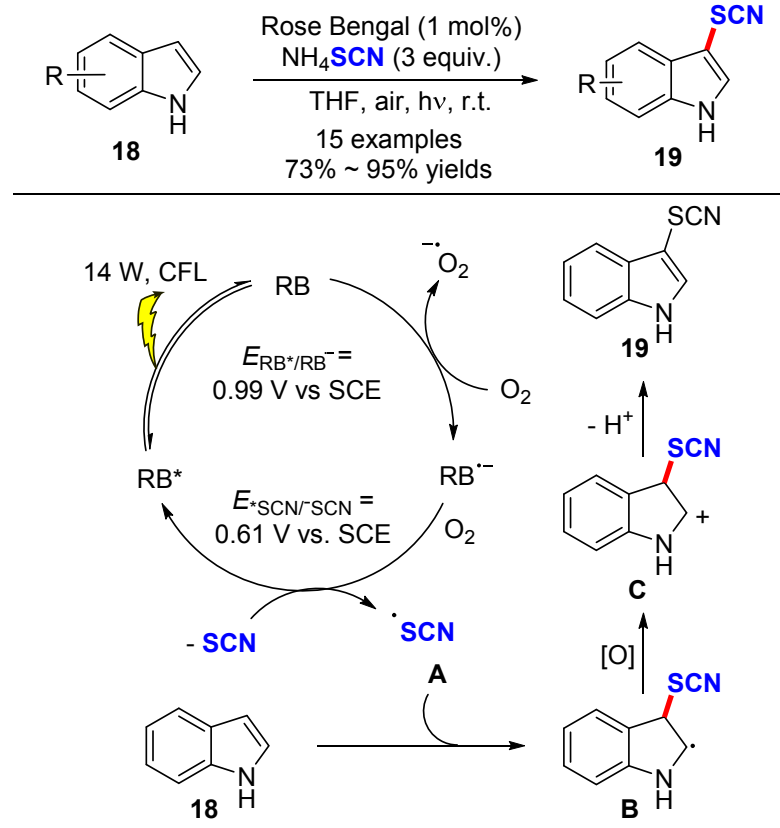

图式 8 可见光催化吲哚的硫氰化反应

Scheme 8 Visible light catalyzed thiocyanation of indoles

范围上还存在一定的局限性, 底物中有硝基或羒基等强 吸电子时, 目标产物的产率较低, 甚至是 N-Boc 保护的 底物仅能获得痕量的产物. 但是, 催化剂的可重复利用 性是该方法的一大优势(Scheme 9).

2018 年, Sarvari 课题组 ${ }^{[15]}$ 通过简单的水热法合成了 一种 $\mathrm{Ag} / \mathrm{TiO}_{2}$ 纳米管为光催化剂, 以空气为终端氧化剂, 通过可见光照射成功地实现了吲哚、苯胺、吡咯和 2氨基噻唑类化合物与硫氰酸铵的硫氰化反应(Scheme 10). 该方法具有底物适用范围宽、光催化剂可循环利 用、催化效率高等优点. 其产物最高收率可达 99\%,为 一系列硫氧化的 $\mathrm{N}$-杂环化合物合成提供新思路.

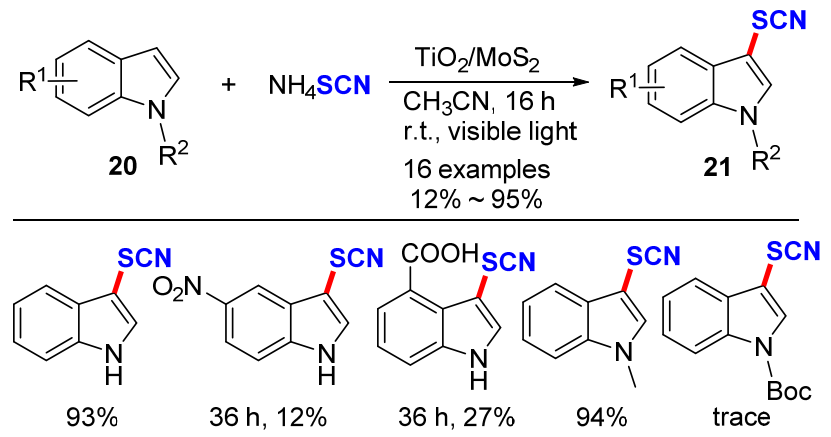

图式 9 可见光促进的 $\mathrm{TiO}_{2} / \mathrm{MoS}_{2}$ 纳米复合物催化吲哚的硫氰 化反应

Scheme 9 Visible-light-promoted $\mathrm{TiO}_{2} / \mathrm{MoS}_{2}$ nanocomposite catalyzed thiocyanation of indoles

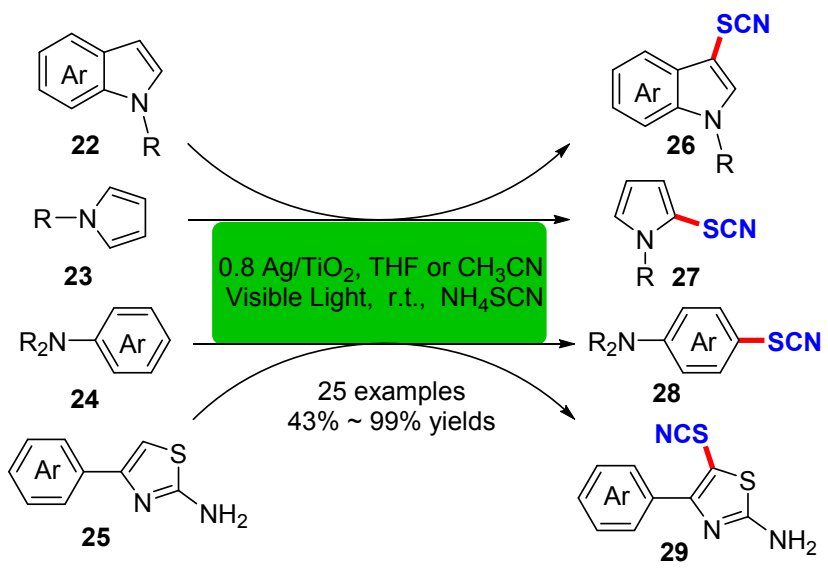

图式 10 可见光促进 $\mathrm{Ag} / \mathrm{TiO}_{2}$ 纳米管催化 $N$-杂环化合物的硫 氧化反应

Scheme 10 Visible-ligh-promoted thiocyanation of $N$-heterocyclic compounds catalyzed by $\mathrm{Ag} / \mathrm{TiO}_{2}$ nanotubes

2015 年, 我们课题组 ${ }^{[16]}$ 以 $\mathrm{KSCN}$ 为硫氰基源, 利用 廉价易得的 $\mathrm{K}_{2} \mathrm{~S}_{2} \mathrm{O}_{8}$ 为氧化剂, 1,2-二氯乙烷(DCE)为溶 
剂, 室温环境下, 成功实现了咪唑并 $[1,2-a]$ 吡啶类化合 物 C-3 位硫氰基化反应(Scheme 11a). 随后, Hajra 课题 组 ${ }^{[17]}$ 利用蓝光照射 Eosin $Y$ 的光催化策略也实现了咪唑 并 [1,2- $a$ 吡啶类化合物 $\mathbf{3 0}$ 的 C-3 位硫氧基化反应 (Scheme 11b). 通过对比两种策略不难发现, 两种方法 都能够以高产率合成具有潜在生物活性的 3-硫氰基咪 唑并 [1,2- $a$ ] 吡啶类化合物 31, 从其底物扩展得知: 无论 是供电子基还是强吸电子基团的底物, 都能很好地适应 两种催化体系.

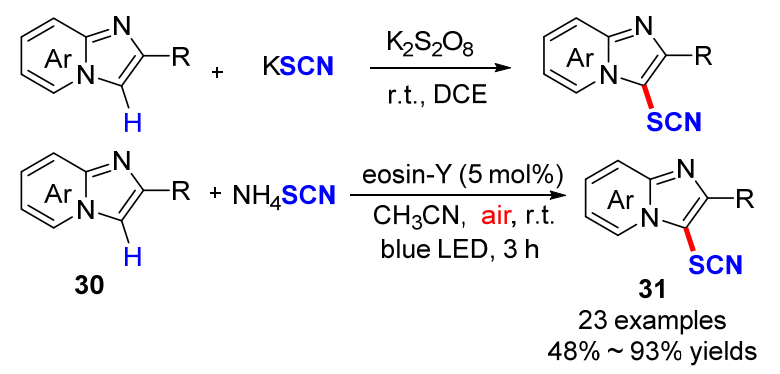

图式 11 咪唑并 $[1,2-a]$ 吡啶类化合物 C-3 位硫氰基化反应 Scheme 11 C-3 thiocyanation of imidazo[1,2-a]pyridines

同年, Singh 课题组 ${ }^{[18]}$ 报道了利用光催化实现了异 腈类衍生物 32 分子内环化硫氧化构建 6-硫氧菲啶类化 合物. 该反应以 $\operatorname{Eosin} \mathrm{Y}$ 为光催化剂, $\mathrm{NH}_{4} \mathrm{SCN}$ 为硫氰基 源, 乙腈反应溶剂, 在室温环境下, 绿光照射 $8 \sim 16 \mathrm{~h}$ 即 可高效合成 17 个带有不同官能团的 6-硫氰菲啶衍生物 33, 产物的收率最高可达 $94 \%$ ，这为 6-硫氰菲啶化合物 的有效合成提供了新思路(Scheme 12).

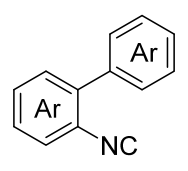

32

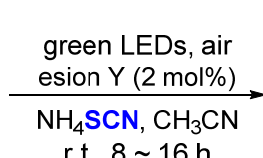

r.t., $8 \sim 16 \mathrm{~h}$

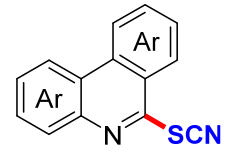

33

17 examples $53 \% \sim 94 \%$ yields
图式 12 光催化合成 6-硫氰菲啶衍生物

Scheme 12 Photocatalytic synthesis of 6-thiophenanthridine derivatives

最近, Jain 等 ${ }^{[19]}$ 报道了以 Eosin $\mathrm{Y}$ 作为可见光催化 剂, 空气作为末端氧化剂, 成功实现了 2-芳基喹啉酮化 合物 34 的 C-3 位硫氰基化反应(Scheme 13). 该方法可 方便快捷地获得多种 2-芳基-3-硫氰基喹诺林-4-酮类化 合物 35. 此外，该方法还可用于选择性实现芳氨类衍生 物 36 对位 $\mathrm{C}-\mathrm{H}$ 键活化硫氰化构建硫氰基芳氨衍生物 37, 且目标产物也有中等至良好的收率.

\section{3 光催化 $\mathrm{C}(\mathrm{sp})-\mathrm{H}$ 的硫氰基化反应}

2018 年, Kshirsagar 课题组 ${ }^{[20]}$ 开发了一种便捷、高 效、无金属的3-硫氰基苯并噻吩的合成方法(Scheme 14).

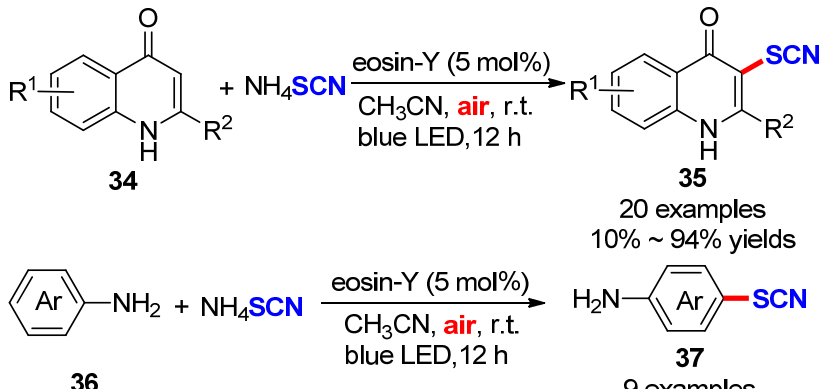

9 examples $45 \% \sim 85 \%$ yields

图式 13 可见光促进的 2-芳基喹啉-4-酮的 C-3 硫氰化反应 Scheme 13 Visible-light-promoted C-3 thiocyanation of 2-arylquinolin-4-ones
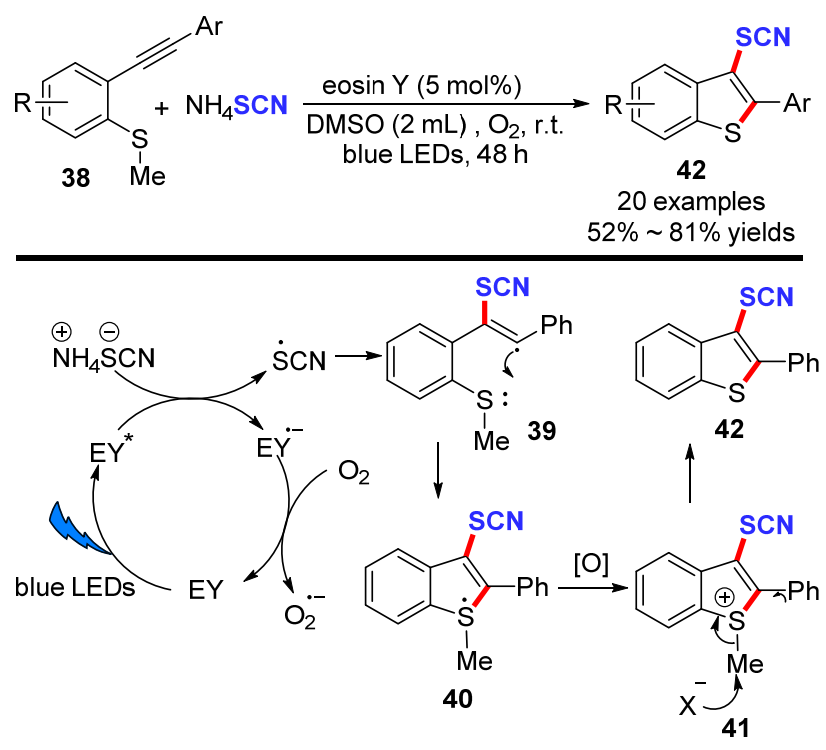

图式 14 可见光催化合成 3-硫氰基苯并噻吩

Scheme 14 Visible light-catalyzed synthesis of 3-thiocyanobenothiophene

该方法以 2-炔基硫代茴香醚化合物 38 作为底物，利用 Eosin Y 作为光敏剂, 在蓝光照射介导下光催化氧化硫 氧酸铵盐的阴离子为硫氧基自由基, 经自由基加成串联 环化得到目标产物 42. 采用这种光催化策略成功实现 了 20 种具有潜在生物活性的 3-取代苯并噻吩衍生物的 绿色合成. 作者根据机理实验和以往文献报道, 提出了 一种可能的机理: 首先, SCN 通过单电子转移过程被激 发态光催化剂 $\mathrm{EY} *$ 氧化为硫氰基自由基, 同时生成 $\mathrm{EY}^{0^{-}}, \mathrm{EY}^{\circ-}$ 被氧气氧化为 $\mathrm{EY}$, 实现催化剂再生, 生成超 氧自由基负离子. 与此同时, 硫氰基自由基与底物 38 作 用经自由基加成串联环化得到中间体 41. 随后, 在 DMSO 作为亲核试剂环境下, 中间体 $\mathbf{4 1}$ 经 $\mathrm{C}-\mathrm{S}$ 断裂脱 甲基过程得到目标产物 $\mathbf{4 2}$.

随后, Gullapalli 等 ${ }^{[21]}$ 采用光催化策略, 成功实现了 末端炔烃 $(43)$ 或联烯 $(\mathbf{4 4})$ 类化合物选择性硫氰化反应构 
建 $\alpha$-硫氧基酮(45)或醇(46). 该方法是以呋啶盐作为光 敏剂, 空气为氧化剂, $\mathrm{NH}_{4} \mathrm{SCN}$ 为硫氰基源, 经白光照 射 6 24 h, 以 15\% 94\%的产率实现 45 个目标化合物 的构筑(Scheme 15).

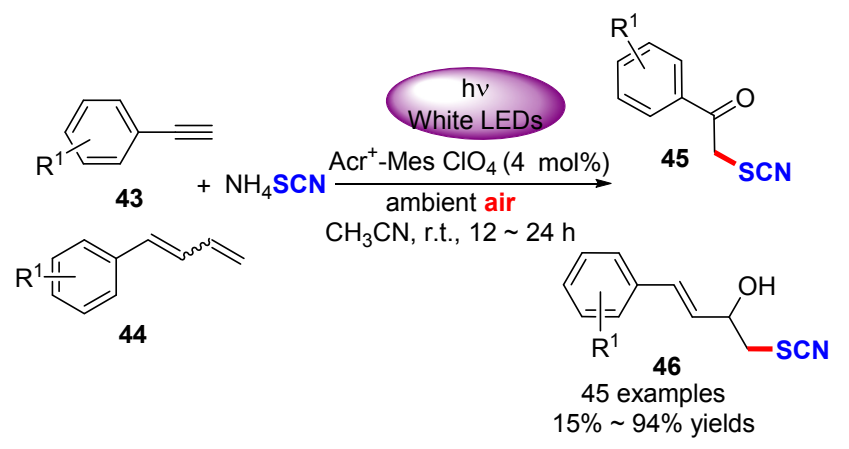

图式 15 可见光诱导合成 $\alpha$-硫氰基酮/硫氰基醇

Scheme 15 Visible light induced synthesis of aryl $\alpha$-thiocyano ketones/thiocyano alcohols

\section{2 电催化硫萱基化}

过去几十年以来, 氧化交叉偶联已被发展成为构建 新化学键最直接的方法之一[22]. 选择廉价易得的底物 作为起始原料, 具有无需预官能化、缩减了繁琐的合成 步骤的优点. 然而, 通常需要氧化剂促进氧化交叉偶联 的进行, 造成了材料成本高, 降低了整体转化的原子经 济性. 此外, 化学计量的氧化剂的使用很有可能引起一 些不必要的副反应或过氧化. 因此, 非常有必要发展无 化学氧化剂参与的氧化偶联反应策略.

近十年来，电化学有机合成作为一种高效、绿色、 环境友好的构筑平台受到了广大有机合成化学家们的 持续关注 ${ }^{[6,22]}$. 电合成是利用电子作为氧化剂, 可避免 化学计量的化学氧化剂的使用, 从而避免了一些化学废 料的生成. 尤其是近几年, 一些电化学氧化交叉偶联反 应被用于 $\mathrm{C}-\mathrm{H}$ 键活化硫氰化构建 $\mathrm{C}-\mathrm{S}$ 键 ${ }^{[220]}$.

\section{1 电催化 $\mathrm{C}\left(\mathrm{sp}^{3}\right)-\mathrm{H}$ 的硫氰基化}

众所周知, 由于 $\mathrm{C}\left(\mathrm{sp}^{3}\right)-\mathrm{H}$ 键的活化需要较高的活 化能, 使得 $\mathrm{C}\left(\mathrm{sp}^{3}\right)-\mathrm{H}$ 键活化要难于 $\mathrm{C}\left(\mathrm{sp}^{2}\right)-\mathrm{H}$. 因此, 该类型的反应受到有机化学家们广泛关注. 例如, 2016 年曾程初课题组 ${ }^{[23]}$ 开发了一种成对电合成反应活化 $\mathrm{C}-\mathrm{H}$ 键, 通过连续的 $\mathrm{C}-\mathrm{S} 、 \mathrm{C}-\mathrm{N}$ 键的形成构建 3-氨 基-2-硫氰基- $\alpha, \beta$-不饱和羰基衍生物(Scheme 16). 作者 利用 $50 \mathrm{~mol} \%$ 的 $\mathrm{NH}_{4} \mathrm{Br}$ 作为电催化媒介, 以铂片作为工 作电极和对电极, 在无外加电解质的乙腈为溶剂中, 以 电流密度为 $5 \mathrm{~mA} / \mathrm{cm}^{2}$ 电解即可以 $11 \% \sim 89 \%$ 的收率获 得 17 个不同类型的 3-氨基-2-硫氰基- $\alpha, \beta$-不饱和羰基衍 生物(48). 在该成对电解过程中, 氨基和硫氰酸酯分子 的来源于单一试剂或乙酸铵和异氧酸钾的组合.

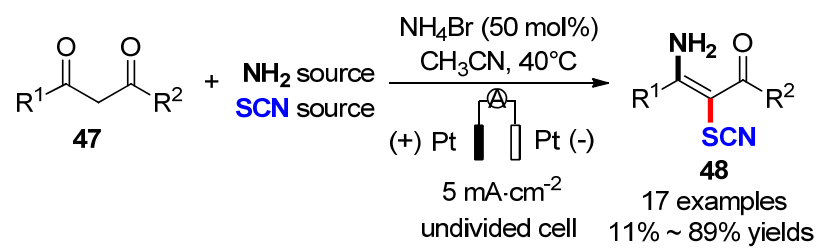

图式 $16 \alpha, \beta$-不饱和羰基酮的硫氰化反应

Scheme 16 Thiocyanation of $\alpha, \beta$-unsaturated carbonyl ketones

随后，他们课题组 ${ }^{[24]}$ 又发展了一种非均相固体盐 Amberlyst-15(H) $[\mathrm{A}-15(\mathrm{H})]$ 为质子催化剂, 以 $\mathrm{NaI}$ 作为电 催化媒介, 采用恒电流电解的方法实现了酮羰基 $\alpha$-位 $\mathrm{C}\left(\mathrm{sp}^{3}\right)-\mathrm{H}$ 键的硫氰基化反应构建硫氰化酮衍生物。与 以往的合成方法相比, 这种方法无需外加电解质. 值得 一提的是，这种新型的固体盐可多次重复使用，且产率 没有显著的降低(Scheme 17).

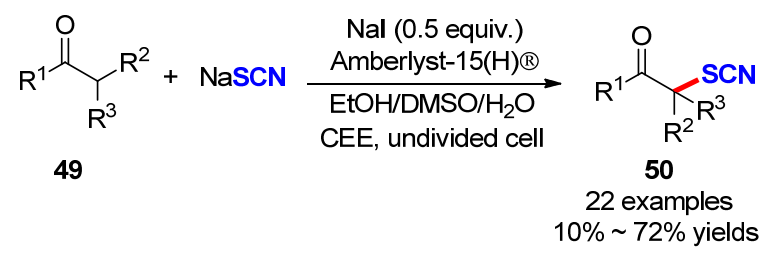

图式 17 酮 $\alpha$ 位硫氰化反应

Scheme $17 \alpha$-Thiocyanation of ketones

\section{2 电催化 $\mathrm{C}\left(\mathrm{sp}^{2}\right)-\mathrm{H}$ 的硫氰基化}

\subsection{1 电催化烯烃的硫氧基化}

电催化烯烃硫氰基化反应的研究相对较早, 在 1973 年 De Klein ${ }^{[25]}$ 利用电氧化硫氰酸盐生成硫氰与烯 烃的加成反应即可获得双硫氰化产物 52. 他们总结了 电氧化烯烃的硫氧化反应, 相对于传统化学合成路径具 有以下优势: (1)双键不可能卤化(当双键与硫氰反应缓 慢，与 $\mathrm{Cl}$ 或 $\mathrm{Br}$ 反应快速时，这特别有利); (2)通过控制 氧化电位或电流, 从而减少副产物的生成. 作者使用恒 电流电解的方法, 在 5 10 ${ }^{\circ} \mathrm{C}$ 的温度的乙酸环境下, 以 $16 \% \sim 80 \%$ 的收率获得一系列新颖的二硫氰酸盐产物， 为电催化硫氧化反应的研究奠定了理论基础(Scheme 18).

2015 年, Becker 课题组 ${ }^{[26]}$ 采用 “阳极一锅法” 在水 和二氯甲烷的两相酸性环境下实现了多取代烯烃硫氧 基化和异硫氰基化反应(Scheme 19). 作者以四甲基乙 烯为模板底物，优化考察了实验涉及到不同的酸、阳极、 电流密度、电荷消耗和电解方法对反应的影响. 研究发 现在两相介质中，以中等产率得到了两个主要产物 $\mathbf{5 4}$ (major)和 55. 其他烷基取代烯烃也产生了类似的加成 产物, 但主要产物是两个 SCN 加成的产物. 然而, 当苯 乙烯和狮酮类底物在水-乙腈组成的均相介质中反应时, 


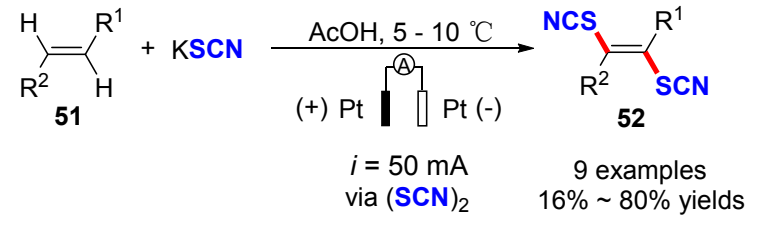

图式 18 烯烃的双硫氰化反应

Scheme 18 Dithiocyanation of olefins
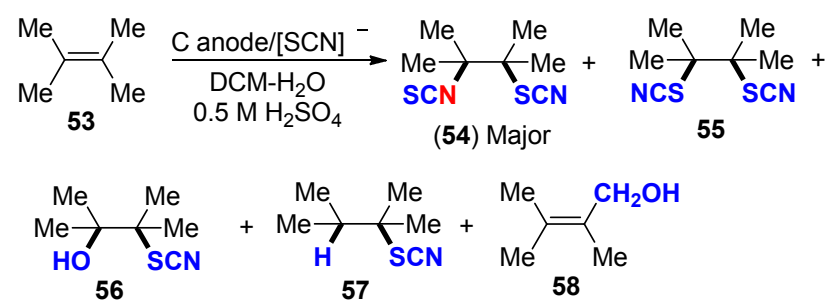

图式 19 四取代烯烃的硫氰化反应

Scheme 19 Thiocyanation of polysubstituted olefins

产物则主要是 $\mathrm{SCN} / \mathrm{OH}$ 加合物.

次年 Becker 课题组 ${ }^{[27]}$ 还开发了一种两步阳极氧化 策略, 实现了烯烃的硫氰化反应(Scheme 20). 首先, 作 者以铂片作为阳极, 钢片作为对电极, $\mathrm{Ag} / \mathrm{AgCl}$ 作为参 比电极, 在以甲酸为溶剂, $\mathrm{LiClO}_{4}$ 为电解质的分离池中 加入 $\mathrm{NH}_{4} \mathrm{SCN}$ 作为硫氧基源, 在电压为 $1.25 \mathrm{~V}$ 的恒电压 模式下经阳极氧化形成硫氰 $(\mathrm{SCN})_{2}$. 随后, 加入不同种 类的烯烃衍生物，经自由基反应路径即可获得目标产物 60.

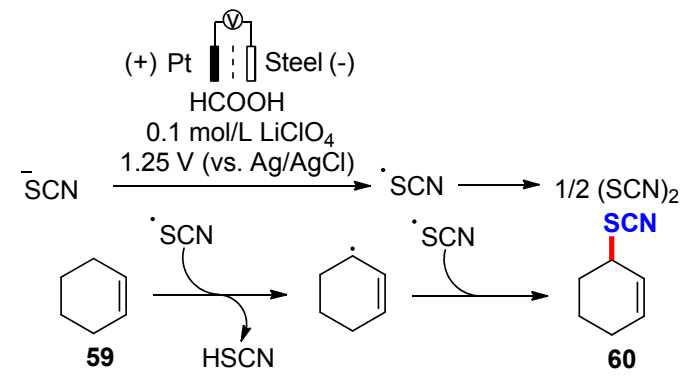

图式 20 烯烃的硫氧化反应

Scheme 20 Thiocyanation of olefins

烯烃 $\mathrm{C}-\mathrm{H}$ 键活化官能化一直是广大合成化学家关 注的热点, 特别是内烯 $\mathrm{C}-\mathrm{H}$ 键活化官能化反应途径成 为多样取代烯烃最直接有效的构建方法之一. 如何实现 便捷、高效、可控性强、环境友好的内烯 $\mathrm{C}-\mathrm{H}$ 键活化 官能化呢? 2019 年, 我们课题组 ${ }^{[28]}$ 报道了一种电化学氧 化条件下 $\mathrm{H}_{2} \mathrm{O}$ 控制内烯选择性硫氧化和烯基化反应. 这 是首例结合电化学 $-\mathrm{H}_{2} \mathrm{O}$ 控制来实现内烯选择性硫氰基 化和烯基化的反应(Scheme 21). 该反应以廉价易得的 二硫代缩醛为原料, 以 $\mathrm{Pt}$ 作为阳极, 碳棒作为阴极, $\mathrm{LiClO}_{4}$ 为电解质, 乙腈为溶剂, 在恒电流常温反应条件 下，通过 $\mathrm{H}_{2} \mathrm{O}$ 控制反应的选择性，实现了内烯的硫氧化 和烯烃的自偶联反应. 结果表明，该体系具有底物适用 范围广和官能团兼容性好等突出优点, 芳基、烷基以及 杂环取代的二硫代缩醛类化合物均能在该反应条件下

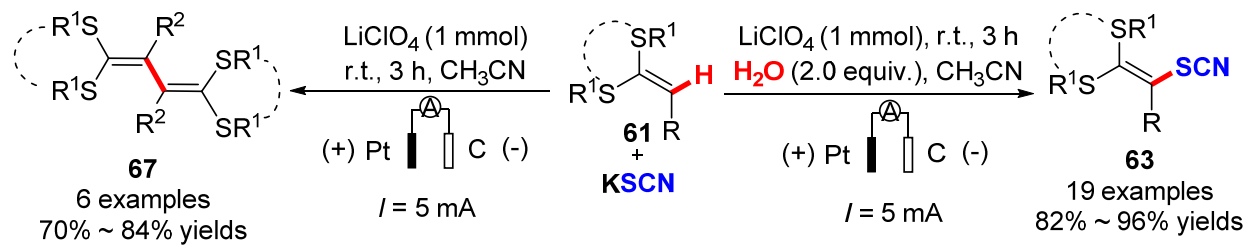

图式 21 烯烃的硫氧化和烯基化反应

Scheme 21 Thiocyanation and alkenylation of alkenes

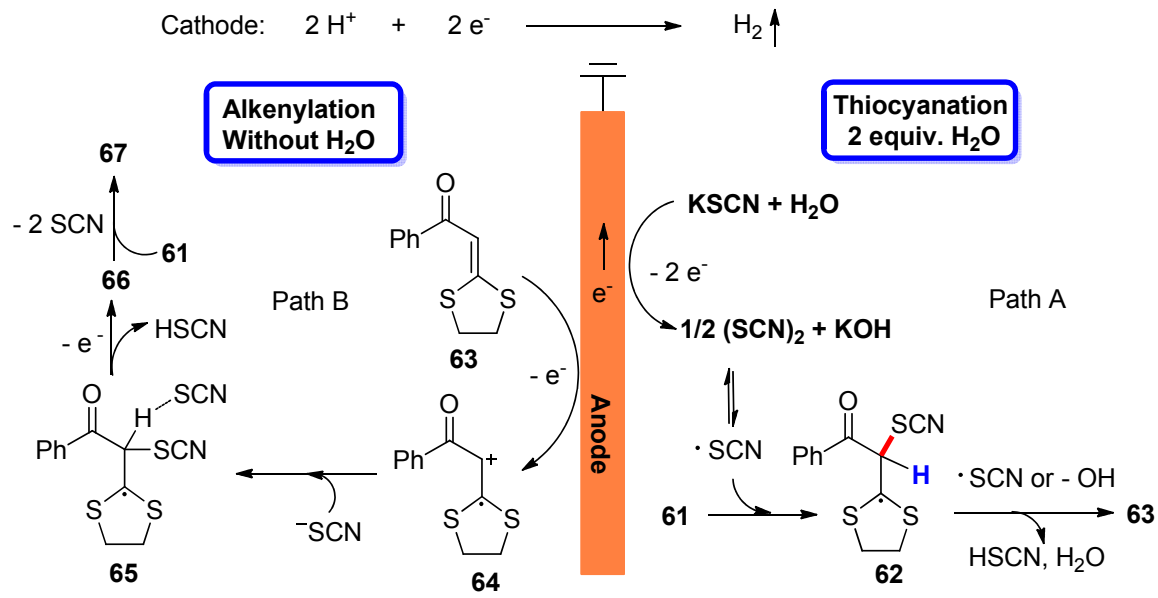

图式 22 烯烃的硫氰化和烯基化反应的机理

Scheme 22 Mechanism of thiocyanation and alkenylation of alkenes 
高选择性地制得相应的硫氰基化产物. 为了深入探究反 应机理, 课题组基于相关文献研究背景, 通过一系列控 制实验以及结合循环伏安测试结果提出了涉及的反应 机理途径. 途径 A: 当水存在时, $\mathrm{KSCN}$ 和水在阳极更容 易被氧化生成 $(\mathrm{SCN})_{2}$, 通过 $(\mathrm{SCN})_{2}$ 均裂得到硫氰基自由 基, 进而与底物发生自由基加成反应获得硫氰化产物 63. 途径 B: 无水条件下, 硫代缩醛底物在阳极失去一 个电子被氧化生成自由基正离子 64 , 与碱性亲电试剂 $\mathrm{KSCN}$ 反应得到中间体 65 , 通过中间体 65 失去一个电 子和质子生成氧基化物 $\mathbf{6 3}$, 最后, 硫氰根作为拟卤素基 团离去获得自偶联产物 67, 同时在阴极放出氢气 (Scheme 22).

2.2.2 电催化芳香族化合物 $\mathrm{C}\left(\mathrm{sp}^{2}\right)$ - $\mathrm{H}$ 活化硫氧基化 硫氰基衍生物是有机合成中很有价值的中间体, 特 别是在杂环化合物的制备方面. 在均相非水溶剂中电解 的缺点是能耗高, 需要纯溶剂和昂贵的辅助电解质盐. 1992 年, Gurjar 课题组 ${ }^{[29]}$ 采用一种两相电解硫氰酸铵生 成 $(\mathrm{SCN})_{3}{ }^{-}$的策略, 成功实现了苯胺、苯酚类衍生物选择 性硫氧化反应(Scheme 23). 随后, 他们课题组还详细研 究了电解温度、电解时间、非水溶剂性质、非水相体积 分数等实验参数对电流效率的影响, 循环伏安测试研究 表明(SCN) ${ }_{3}{ }^{-}$是产生于水相体系中后被带入非水相中参 与反应 ${ }^{[30]}$.

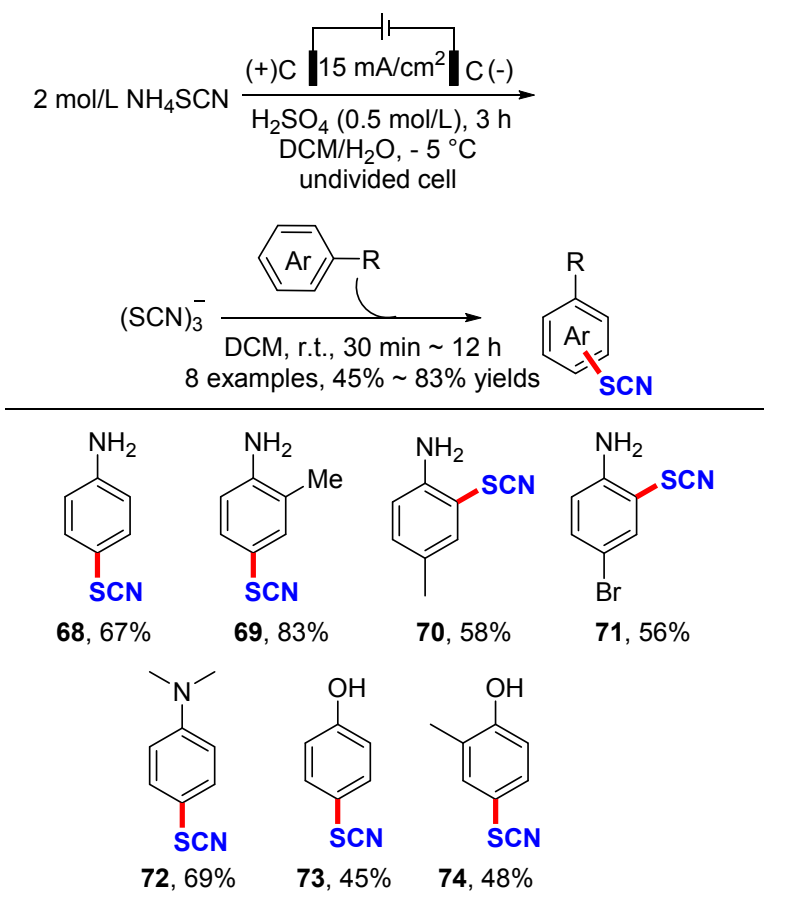

图式 23 芳胺和苯酚类衍生物的硫氰化反应

Scheme 23 Thiocyanation of aniline and phenol derivatives

芳香硫氰酸盐是制备杂环化合物的重要中间体，特 别是用作农用化学品、染料和药品的前体. 在各种已知
的硫氰化方法中，以硫氰 $(\mathrm{SCN})_{2}$ 作为硫氰化剂最为常 见. 然而, 由于 $(\mathrm{SCN})_{2}$ 对水、光和热的敏感性, 以及在 非水介质中易于聚合，其稳定性较差，因此，其取代产 物的产率通常较低. 基于此, Becker 课题组 ${ }^{[31]}$ 在 2006 年 采用电化学方法成功实现了苯甲醚对位硫氰化反应 (Scheme 24). 作者以苯甲醚 75 为底物对比性地研究了 恒电流、恒电压电解的区别, 考察了阳极材料、溶剂、 耗电量、电流密度、电解质和浓度等因素对硫氧基化产 物 76 形成的影响.

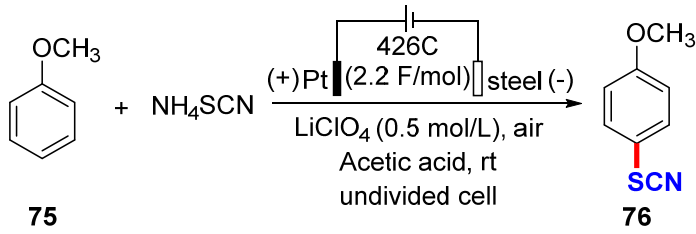

图式 24 茴香醚的电化学硫氰化反应

Scheme 24 Electrochemical thiocyanation of anisole

2010 年, Becker 课题组 ${ }^{[32]}$ 又利用 H-型的分离电解 池在恒电压的酸性条件下, 选择性地实现了苯甲醚、甲 苯和苯胺类衍生物对位硫氰基化反应(Scheme 25). 作 者认为该反应首先是硫氰酸根在阳极被氧化生成硫氰 基自由基，硫氰基自由基发生聚合得到硫氰( $\mathrm{SCN})_{2}$. 随 后， $(\mathrm{SCN})_{2}$ 被酸性溶剂极化，并攻击底物的芳香核以提 供相应的硫氰酸酯衍生物 78. 但遗憾的是, 该方法对芳 香胺类底物收率较低，仅有 $15 \%$ 42\%的产率.

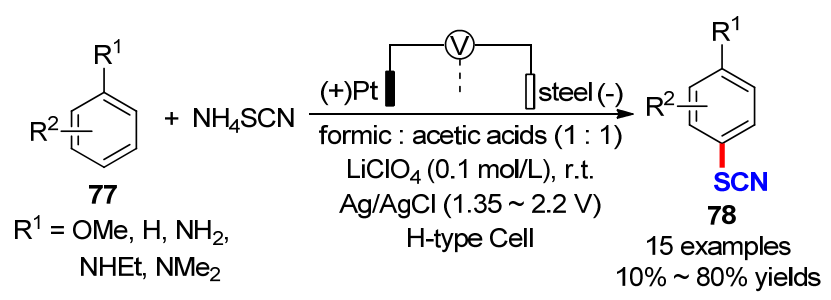

图式 25 取代茴香醚、甲苯和苯胺衍生物的电化学硫氰化反 应

Scheme 25 Electrochemical thiocyanation of substituted anisole, toluene and aniline derivatives

\subsection{3 电催化芳杂环的 $\mathrm{C}\left(\mathrm{sp}^{2}\right)-\mathrm{H}$ 活化硫氧基化}

硫氰酸盐基团在化学和生物化学上具有广泛的用 途，已报道了多种芳族的硫氰化方法. 例如 $\mathrm{I}_{2} / \mathrm{NH}_{4} \mathrm{SCN}$, $\mathrm{Mn}(\mathrm{OAc})_{3} / \mathrm{NH}_{4} \mathrm{SCN}$, 酸性的蒙脱石 (K-10), $\mathrm{Zn}(\mathrm{SCN})_{2} /$ $\mathrm{Cl}_{2}, \mathrm{H}_{5} \mathrm{IO}_{6} / \mathrm{KSCN}, \mathrm{FeCl}_{3}$, 硝酸铈铵, 三氟异氭尿酸 $/ \mathrm{SiO}_{2}$ 等 ${ }^{[33]}$. 然而, 这些方法中存在一些缺点, 包括产率低(特 别是芳香胺的硫氰化)、强酸性或苛刻的氧化条件、反 应时间长和/或高温以及使用昂贵和有毒的试剂. 基于 此, 2013 年 Nikoofar 课题组 ${ }^{[34]}$ 报道了一种含氮(杂)芳香 族化合物在恒电流条件下, 以硫氰酸铵为原料, 在室温 
条件下进行阳极硫氰化反应. 值得指出的是, 吲哚、咔 唑、二苯胺类型的 $\mathrm{N}$-杂原子底物在该反应条件下都能以 中等的收率得到目标产物 $79 \sim 85$. 此外, 利用该策略以 二苯胺为原料还能得到双硫氰化的产物 86 (Scheme 26).

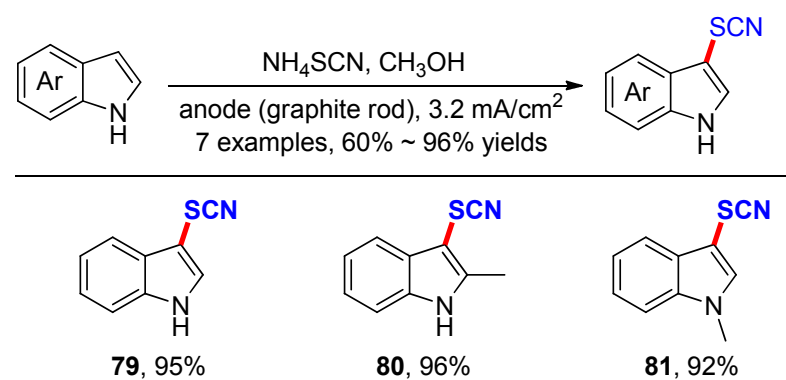<smiles>CC(C)(C)O[R6](=O)(O[Na])Oc1ccc2c(c1)C(=O)C(=O)N2</smiles><smiles>N#Sc1ccc(Nc2ccc(S(N)(=O)=O)cc2)cc1</smiles>

$85,60 \%$

86, $10 \%$

图式 26 含氮杂环的电化学硫氰化反应

Scheme 26 Electrochemical thiocyanation of nitrogen-containing heterocycles

2014 年 Petrosyan 课题组 ${ }^{[35]}$ 在 path I 的基础上, 通 过阳极氧化硫氰酸根生成硫氰，与芳烃发生亲核取代反 应(path II b), 成功实现了吲哚、 $N$-甲基吡咯、吡唑和苯 胺等 $N$-杂环衍生物的硫氰化反应(Scheme 27). 值得注 意的是, 相比于 Becker 教授的工作, 该方法对于选择性 实现苯胺对位硫氰化反应构建化合物 90 具有更高的收 率 $(75 \%)$.

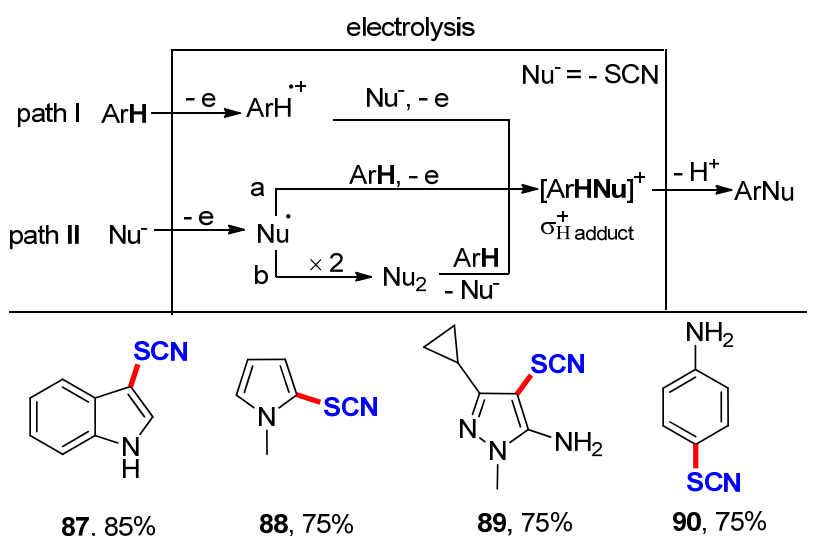

图式 $27 N$-杂芳烃衍生物的电化学硫氧化反应 Scheme 27 Electrochemical thiocyanation of $N$-heteroaromatic derivatives

2018 年, 江洪课题组 ${ }^{[36]}$ 开发了一种低成本、高效的 富电子芳烃硫代和硒代㲵化策略(Scheme 28). 在没有
任何催化剂或氧化剂的温和条件下，这种策略适用于各 种情况(杂)芳香族化合物，如吲哚、吡咯、苯胺和茴香 醚等衍生物的硫氧基化和硒基化反应，以 52\% 99\%的 产率实现 45 个不同取代基的硫代和硒代氰化芳杂环化 合物的构建. 遗憾的是, 该方法不能得到硒氰化的均三 苯甲醚产物 97 和硒氰化的 $N$-甲基吡咯产物 99, 能够得 到双硫氰化产物 100, 但产率较低. 2019 年, Petrosyan 课 题组 ${ }^{[37]}$ 在此基础上，提出了一种两步控制电压电解的 策略，成功实现了 $N$-甲基吡咯的双 $\mathrm{C}-\mathrm{H}$ 键活化硫氰化， 产率提升到了 $52 \%$ (Scheme 29).
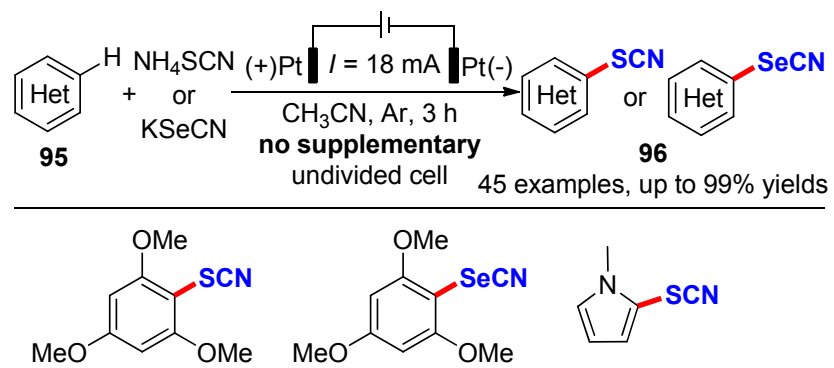
96, $97 \%$<smiles>Cn1cccc1SC#N</smiles>

99, $0 \%$

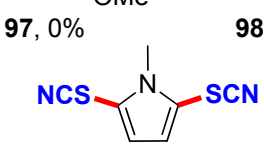

$100,15 \%$
图式 28 富电子芳烃的硫代和硒代氰化策略

Scheme 28 Thio- and seleno-cyanation strategies for electron-rich arenes

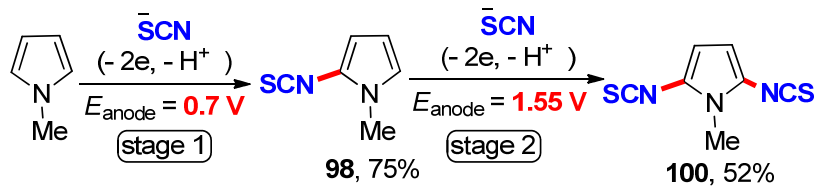

图式 $29 \mathrm{~N}$-甲基吡咯的阳极双 $\mathrm{C}-\mathrm{H}$ 硫㲵化反应 Scheme 29 Double anodic $\mathrm{C}-\mathrm{H}$ thiocyanation of $N$-methylpyrrole

卤代吲哚作为生物活性的天然产物中必不可少的 结构单元, 受到了合成化学家们极大关注. 江洪课题 组 ${ }^{[38]}$ 同样在没有任何催化剂或氧化剂, 无额外电解质 的温和条件的电化学策略, 成功实现哚类衍生物 C-3 位卤化 $(\mathrm{Cl}, \mathrm{Br}, \mathrm{I})$ 或硫氰化反应, 各种含电子丰富的吲哚 衍生物表现出了很大的转化效率(Scheme 30).

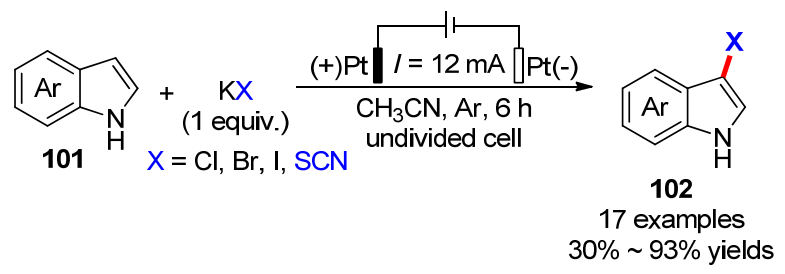

图式 30 吲哚衍生物的卤化和硫氰化反应

Scheme 30 Halogenation and thiocyanation of indole derivatives 
2016 年, Kokorekin 教课题组 ${ }^{[39]}$ 采用电化学方法, 在室温条件下以 $\mathrm{Pt}$ 作为阳极, $\mathrm{NH}_{4} \mathrm{SCN}$ 作为硫氰基源, $\mathrm{NaClO}_{4}$ 作为电解质, $\mathrm{CH}_{3} \mathrm{CN}$ 作为溶剂, 实现了吡唑并 [1,5- $a$ ] 嘧啶化合物 103 的 3 位选择性硫氰酸化反应, 产 物 104 的收率为 $69 \% \sim 87 \%$. 但是对于具有较高氧化电 位的底物, 当作者在其反应体系中加入 $\mathrm{ZnCl}_{2}$ 时, 其收 率可从 $0 \%$ 提高到 $70 \%$. 作者认为 $\mathrm{ZnCl}_{2}$ 起着路易斯酸的 作用. 他们的研究揭示了电氧化吡唑并 $[1,5-a]$ 嘧啶硫氧 酸化的反应的效率取决于底物的氧化电位, 路易斯酸的 加入可以帮助调节底物的氧化电势(Scheme 31).

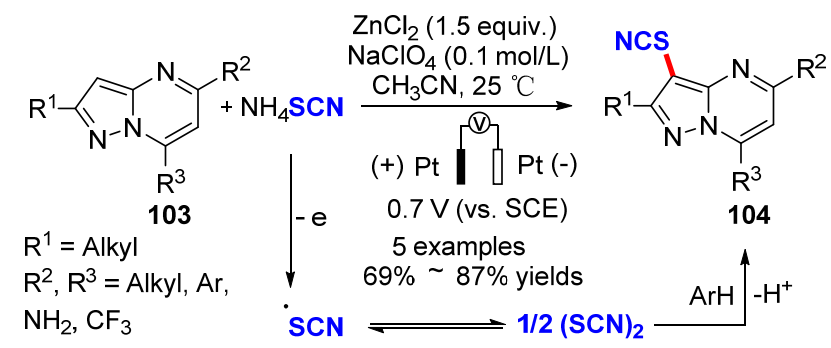

图式 31 吡唑并 $[1,5-a]$ 嘧啶的硫氰酸化反应

Scheme 31 Thiocyanation of pyrazoline $[1,5-a]$ pyrimidine

随后, 他们课题组 ${ }^{[40]}$ 分别针对高活性和低活性的 吡唑并 $[1,5-a]$ 嘧啶衍生物的电氧化硫氰化反应开发三种 新的互补体系(32). 第一种方法(电氧化-SCN 生成 $\left.(\mathrm{SCN})_{2}\right)$ 适用于高活性芳烃 $\left(E_{\mathrm{p}}<1.70 \mathrm{~V}\right)$, 第二种方法(芳 基阳离子生成法)和第三种方法 $\left[\mathrm{ZnCl}_{2}\right.$, via $\left.(\mathrm{SCN})_{2}\right]$ 适用 于低反应性的芳烃 $\left(E_{\mathrm{p}}>1.70 \mathrm{~V}\right)$. 作者以硫氰酸盐 $\left(\mathrm{NH}_{4} \mathrm{SCN}\right.$ 或 $\left.\mathrm{KSCN}\right)$ 为硫氰基源, 在室温下电解硫氧酸 根离子/吡唑并 $[1,5-a$ ]嘧啶 105 混合物, 成功地实现了 11 种具有抗真菌活性的 3-硫氰酸基吡唑并 $[1,5-a$ ] 嘧啶衍生 物 106 的合成，其目标产物的产率为 $60 \% \sim 89 \%$ (Scheme 32).

$$
\text { (+) } \mathrm{Pt} \prod \mathrm{Ht}(-)
$$

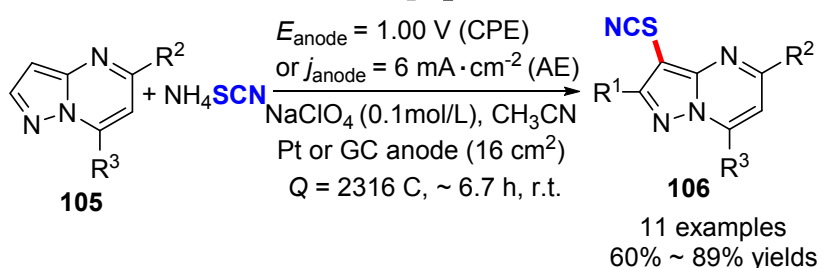

图式 32 吡唑并 $[1,5-a]$ 嘧啶的硫氰酸化反应

Scheme 32 Thiocyanation of pyrazoline $[1,5-a]$ pyrimidine

\section{3 电催化硼酸类和肉桂酸化合物的硫氭基化}

2019 年, Gooßen 等 ${ }^{[41]}$ 提出了一种简单的、无过渡金 属催化的异硫氧化芳基嗍酸和三氟硼酸芳酯构建芳基 硫氰化物的电化学方法. 硫氰酸盐阴离子经阳极氧化原
位生成 $\mathrm{SCN}$ 亲电试剂, 以高到 $100 \%$ 的产率实现了 22 个不同类型的芳基硼酸衍生物的硫氰基化反应. 遗憾的 是，带有强吸电子基团类芳基硼酸和烷基硼酸类底物在 该反应条件下不能得到产物(Scheme 33).

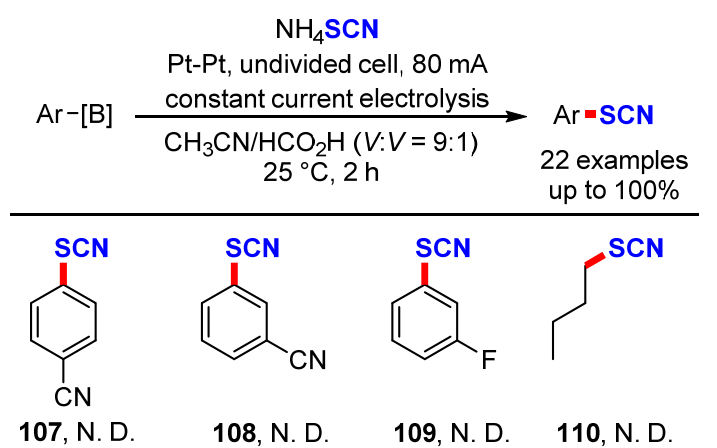

图式 33 芳基硼酸和三氟嗍酸芳基的电化学硫氰化反应 Scheme 33 Electrochemical thiocyanation of arylboronic acids and aryl trifluoroborates

同年, Huang 课题组 ${ }^{[42]}$ 利用 Kolbe 反应，报道了一 种简单、绿色的电催化肉桂酸 111 与 $\mathrm{NH}_{4} \mathrm{SCN}$ 在水溶液 中脱翔偶联制备芳基乙烯硫氰酸酯的电化学方法 (Scheme 34). 采用恒电流电解的方法, 以 $\mathrm{NH}_{4} \mathrm{SCN}$ 为硫 氰基源，乙腈和水作为溶剂, $\mathrm{NaHCO}_{3}$ 作为碱，高氯酸钠 作为电解质，室温 $5 \mathrm{~mA}$ 电解反应 $9 \mathrm{~h}$, 以 $12 \% \sim 80 \%$ 产 率合成 22 种芳基乙烯硫氰酸酯化合物. 但不足的是，带 有强吸电子基团的烯烃作为底物时产率相对较低，甚至 烷基烯烃在该反应条件下不能够得到理想的目标产物. 作者对该反应提出了一种可能的反应机理：首先，硫氰 酸根阴离子在阳极表面上经单电子氧化得到硫氰酸根

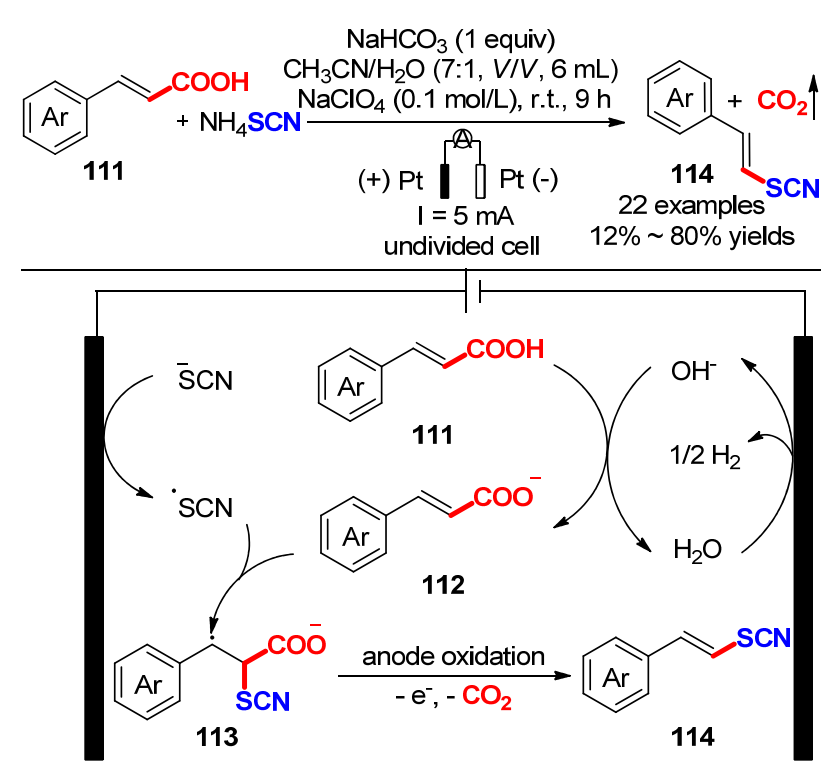

图式 34 电催化脱羧肉桂酸的硫氰化反应

Scheme 34 Electrochemical decarboxylative thiocyanation of cinnamic acids 
自由基中间体. 随后, 生成的硫氰酸根自由基与 112 发 生自由基加成形成中间体 113, 113 经过脱羧得到目标产 物乙烯基硫氰酸酯(114). 但该反应也不能排除阳极氧 化硫氰酸根阴离子生成硫氰 $(\mathrm{SCN})_{2}$ 参与反应的可能性.

\section{3 总结与展望}

近年来, 通过使用可见光、电催化介入的硫氰基化 反应这两种新型、绿色的构筑策略, 可以实现一系列热 力学上难以实现的硫氰基化反应. 这为合成医药、功能 材料等提供了新的方法, 且具有绿色、高效、安全等优 势. 尽管光、电催化介导的硫氰化反应策略已取得了一 些进展，但对于复杂分子和天然产物分子的硫氰化修饰 仍然还处于研究初期. 电化学有机合成是通过电子的 得失来替代传统的氧化还原试剂, 可通过改变电流或电 压及时调控反应活化能、控制反应的选择性, 是一种的 绿色、经济的策略, 可实现一系列化学转化, 极具潜力. 相信该类合成策略能够得到进一步的优化和拓展, 在更 多的催化反应中应用, 适用于更多种类的底物, 为医 药、功能材料领域中硫氰化物的制备提供绿色、高效、 环境友好的平台.

\section{References}

[1] (a) Capon, R. J.; Skene, C.; Liu, E. H.-T.; Lacey, E.; Gill, J. H.; Heiland, K.; Friedel, T. J. Org. Chem. 2001, 66, 7765.

(b) Dutta, S.; Abe, H.; Aoyagi, S.; Kibayashi, C.; Gates, K. S. J. Am. Chem. Soc. 2005, 127, 15004.

(c) Elhalem, E.; Bailey, B. N.; Docampo, R.; Ujváry, I.; Szajnman, S. H.; Rodriguez, J. B. J. Med. Chem. 2002, 45, 3984.

(d) Kokorekin, V.; Terent'ev, A.; Ramenskaya, G.; Grammatikova, N.; Rodionova, G.; Ilovaiskii, A. Pharm. Chem. J. 2013, 47, 422.

(e) Yasman, Y.; Edrada, R. A.; Wray, V.; Proksch, P. J. Nat. Prod. 2003, 66, 1512.

[2] (a) Khalili, D. Chin. Chem. Lett. 2015, 26, 547.

(b) Zeng, Y.-F.; Tan, D.-H.; Chen, Y.; Lv, W.-X.; Liu, X.-G.; Li, Q.; Wang, H. Org. Chem. Front. 2015, 2, 1511.

(c) Guo, L.-N.; Gu, Y.-R.; Yang, H.; Hu, J. Org. Biomol. Chem. 2016, 14, 3098.

(d) Khalili, D. New J. Chem. 2016, 40, 2547.

(e) Chen, Q.; Lei, Y.; Wang, Y.; Wang, C.; Wang, Y.; Xu, Z.; Wang, H.; Wang, R. Org. Chem. Front. 2017, 4, 369.

(f) Ji, F.; Fan, Y.; Yang, R.; Yang, Y.; Yu, D.; Wang, M.; Li, Z. Asian J. Org. Chem. 2017, 6, 682.

(g) Jiang, G.; Zhu, C.; Li, J.; Wu, W.; Jiang, H. Adv. Synth. Catal. 2017, 359, 1208.

(h) Prieto, A.; Uzel, A.; Bouyssi, D.; Monteiro, N. Eur. J. Org. Chem. 2017, 2017, 4201.

(i) Chen, Y.; Wang, S.; Jiang, Q.; Cheng, C.; Xiao, X.; Zhu, G. $J$. Org. Chem. 2018, 83, 716.

(j) Khaikate, O.; Meesin, J.; Pohmakotr, M.; Reutrakul, V.; Leowanawat, P.; Soorukram, D.; Kuhakarn, C. Org. Biomol. Chem. 2018, 16, 8553.

(k) Qiu, J.; Wu, D.; Karmaker, P. G.; Yin, H.; Chen, F.-X. Org. Lett. 2018, 20, 1600 .

(1) Wu, C.; Lu, L.-H.; Peng, A.-Z.; Jia, G.-K.; Peng, C.; Cao, Z.; Tang, Z.; He, W.-M.; Xu, X. Green Chem. 2018, 20, 3683.

(m) Dey, A.; Hajra, A. Adv. Synth. Catal. 2019, 361, 842.

(n) Noikham, M.; Yotphan, S. Eur. J. Org. Chem. 2019, 2019, 2759.
[3] Castanheiro, T.; Suffert, J.; Donnard, M.; Gulea, M. Chem. Soc. Rev. 2016, 45, 494

[4] Vekariya, R. H.; Patel, H. D. Synth. Commun. 2017, 47, 87.

[5] Qin, X.; Zhang, L.-Y.; Feng, G.-F.; Jin, C.-A. Chin. J. Org. Chem. 2019, 39, 287 (in Chinese). (徐庆, 张连阳, 冯高峰, 金城安, 有机化学, 2019, 39, 287.)

[6] (a) Hartwig, J. F. Nature 2008, 455, 314.

(b) Lu, Q.; Zhang, J.; Wei, F.; Qi, Y.; Wang, H.; Liu, Z.; Lei, A. Angew. Chem., Int. Ed. 2013, 52, 7156.

(c) Lu, Q.; Zhang, J.; Zhao, G.; Qi, Y.; Wang, H.; Lei, A. J. Am. Chem. Soc. 2013, 135, 11481.

(d) Xi, Y.; Dong, B.; McClain, E. J.; Wang, Q.; Gregg, T. L.; Akhmedov, N. G.; Petersen, J. L.; Shi, X. Angew. Chem., Int. Ed. 2014, 53, 4657.

(e) Shen, C.; Zhang, P.; Sun, Q.; Bai, S.; Hor, T. A.; Liu, X. Chem. Soc. Rev. 2015, 44, 291.

(f) Liu, Y.; Xiong, J.; Wei, L. Chin. J. Org. Chem. 2017, 37, 1667 (in Chinese).

(刘云云, 熊进, 韦丽, 有机化学, 2017, 37, 1667.)

(g) Mei, H.; Yin, Z.; Liu, J.; Sun, H.; Han, J. Chin. J. Chem. 2019, 37, 292.

(h) Yang, Q.-L.; Fang, P.; Mei, T.-S. Chin. J. Chem. 2018, 36, 338.

[7] Yadav, A. K.; Yadav, L. D. S. Tetrahedron Lett. 2015, 56, 6696.

[8] Yuan, P.-F.; Zhang, Q.-B.; Jin, X.-L.; Lei, W.-L.; Wu, L.-Z.; Liu, Q. Green Chem. 2018, 20, 5464.

[9] Guy, R. G.; Thompson, J. J. Tetrahedron 1978, 34, 541.

[10] Chen, Y.-J.; He, Y.-H.; Guan, Z. Tetrahedron 2019, 75, 3053.

[11] Zhang, D.; Wang, H.; Bolm, C. Chem. Commun. 2018, 54, 5772.

[12] Gao, Y.; Liu, Y.; Wan, J.-P. J. Org. Chem. 2019, 84, 2243.

[13] Fan, W.; Yang, Q.; Xu, F.; Li, P. J. Org. Chem. 2014, 79, 10588.

[14] Wang, L.; Wang, C.; Liu, W.; Chen, Q.; He, M. Tetrahedron Lett. 2016, 57, 1771.

[15] Hosseini-Sarvari, M.; Hosseinpour, Z.; Koohgard, M. New J. Chem. 2018, 42, 19237

[16] Yang, D.; Yan, K.; Wei, W.; Li, G.; Lu, S.; Zhao, C.; Tian, L.; Wang, H. J. Org. Chem. 2015, 80, 11073.

[17] Mitra, S.; Ghosh, M.; Mishra, S.; Hajra, A. J. Org. Chem. 2016, 47, 8275.

[18] Singh, M.; Yadav, A. K.; Yadav, L. D. S.; Singh, R. Synlett 2018, $29,176$.

[19] Chauhan, P.; Ritu, R.; Preeti, P.; Kumar, S.; Jain, N. Eur. J. Org. Chem. 2019, 2019, 4334

[20] Tambe, S. D.; Jadhav, M. S.; Rohokale, R. S.; Kshirsagar, U. A. Eur. J. Org. Chem. 2018, 2018, 4867.

[21] Gullapalli, K.; Vijaykumar, S. Org. Biomol. Chem. 2019, 17, 2232.

[22] (a) Zhao, Y.; Wang, H.; Hou, X.; Hu, Y.; Lei, A.; Zhang, H.; Zhu, L. J. Am. Chem. Soc. 2006, $128,15048$.

(b) Li, C.-J. Acc. Chem. Res. 2009, 42, 335.

(c) Chen, M.; Zheng, X.; Li, W.; He, J.; Lei, A. J. Am. Chem. Soc. 2010, 132, 4101 .

(d) Le Bras, J.; Muzart, J. Chem. Rev. 2011, 111, 1170.

(e) Liu, C.; Zhang, H.; Shi, W.; Lei, A. Chem. Rev. 2011, 111, 1780.

(f) Shi, W.; Liu, C.; Lei, A. Chem. Soc. Rev. 2011, 40, 2761.

(g) Yeung, C. S.; Dong, V. M. Chem. Rev. 2011, 111, 1215.

(h) He, C.; Guo, S.; Ke, J.; Hao, J.; Xu, H.; Chen, H.; Lei, A. J. Am. Chem. Soc. 2012, 134, 5766.

(i) Girard, S. A.; Knauber, T.; Li, C. J. Angew. Chem., Int. Ed. 2014, 53,74 .

(j) Liu, C.; Liu, D.; Lei, A. Acc. Chem. Res. 2014, 47, 3459.

(k) Liu, C.; Yuan, J.; Gao, M.; Tang, S.; Li, W.; Shi, R.; Lei, A. Chem. Rev. 2015, 115, 12138.

(1) Zhang, G.; Liu, C.; Yi, H.; Meng, Q.; Bian, C.; Chen, H.; Jian, J.-X.; Wu, L.-Z.; Lei, A. J. Am. Chem. Soc. 2015, 137, 9273.

(m) Song, C.; Yi, H.; Dou, B.; Li, Y.; Singh, A. K.; Lei, A. Chem. Commun. 2017, 53, 3689.

(n) Song, C.; Dong, X.; Yi, H.; Chiang, C.-W.; Lei, A. ACS Catal. 2018, 8, 2195 .

(o) Song, C.; Liu, K.; Dong, X.; Chiang, C.-W.; Lei, A. Synlett 
2019, 30, 1149 .

[23] Kang, L.-S.; Luo, M.-H.; Lam, C. M.; Hu, L.-M.; Little, R. D.; Zeng, C.-C. Green Chem. 2016, 18, 3767.

[24] Liang, S.; Zeng, C. C.; Tian, H.; Sun, B.; Ren, F. Adv. Synth. Catal. 2018, 360, 1444.

[25] De Klein, W. J. Electrochim. Acta 1973, 18, 413.

[26] Levy, A.; Becker, J. Y. Electrochim. Acta 2015, 178, 294.

[27] Gitkis, A.; Becker, J. Y. Electroanalysis 2016, 28, 2802.

[28] Wen, J.; Zhang, L.; Yang, X.; Niu, C.; Wang, S.; Wei, W.; Sun, X.; Yang, J.; Wang, H. Green Chem. 2019, 21, 3597.

[29] Krishnan, P.; Gurjar, V. G. Synth. Commun. 1992, 22, 2741.

[30] Krishnan, P.; Gurjar, V. G. J. Appl. Electrochem. 1995, 25, 792.

[31] Gitkis, A.; Becker, J. Y. J. Electroanal. Chem. 2006, 593, 29.

[32] Gitkis, A.; Becker, J. Y. Electrochim. Acta 2010, 55, 5854.

[33] (a) Nair, V.; George, T. G.; Nair, L. G.; Panicker, S. B. Tetrahedron Lett. 1999, 40, 1195.

(b) Chakrabarty, M.; Sarkar, S. Tetrahedron Lett. 2003, 44, 8131.

(c) Yadav, J.; Reddy, B.; Shubashree, S.; Sadashiv, K. Tetrahedron Lett. 2004, 45, 2951.

(d) Yadav, J.; Reddy, B.; Krishna, A.; Reddy, C. S.; Narsaiah, A. Synthesis 2005, 2005, 961

(e) Pan, X.-Q.; Lei, M.-Y.; Zou, J.-P.; Zhang, W. Tetrahedron Lett.
2009, 50, 347.

(f) Akhlaghinia, B.; Pourali, A.-R.; Rahmani, M. Synth. Commun. 2012, 42, 1184.

(g) Khazaei, A.; Zolfigol, M. A.; Mokhlesi, M.; Panah, F. D.; Sajjadifar, S. Helv. Chim. Acta 2012, 95, 106.

[34] Fotouhi, L.; Nikoofar, K. Tetrahedron Lett. 2013, 54, 2903.

[35] Kokorekin, V. A.; Sigacheva, V. L.; Petrosyan, V. A. Tetrahedron Lett. 2014, 55, 4306.

[36] Zhang, X.; Wang, C.; Jiang, H.; Sun, L. RSC Adv. 2018, 8, 22042.

[37] Yaubasarova, R. R.; Kokorekin, V. A.; Ramenskaya, G. V.; Petrosyan, V. A. Mendeleev Commun. 2019, 29, 334.

[38] Sun, L.; Zhang, X.; Li, Z.; Ma, J.; Zeng, Z.; Jiang, H. Eur. J. Org. Chem. 2018, $2018,4949$.

[39] Kokorekin, V. A.; Yaubasarova, R. R.; Neverov, S. V.; Petrosyan, V. A. Mendeleev Commun. 2016, 5, 413.

[40] Kokorekin, V. A.; Yaubasarova, R. R.; Neverov, S. V.; Petrosyan, V. A. Eur. J. Org. Chem. 2019, 2019, 4233.

[41] Dyga, M.; Hayrapetyan, D.; Rit, R. K.; Gooßen, L. J. Adv. Synth. Catal. 2019, 361, 3548.

[42] Yang, S.-M.; He, T.-J.; Lin, D.-Z.; Huang, J.-M. Org. Lett. 2019, 21, 1958. 